\title{
X-RAY AND OPTICAL OBSERVATIONS OF BY CAM
}

\author{
GAVIN RAMSAY ${ }^{1}$, PAUL A. MASON ${ }^{2}$
}

1. Astronomical Institute, The University of Utrecht, Postbus 80000, Utrecht, NL 3508 TA, The Netherlands

2. Astronomy Department, Case Western Reserve University, Cleveland, $\mathrm{OH}$ 44106, USA

\section{Introduction}

We present preliminary results of an analysis of X-ray and optical data of the asynchronous AM Her star BY Cam [1]. We use X-ray data from EXOSAT $(0.1 \ldots 50 \mathrm{keV})$, Ginga $(1.5 \ldots 50 \mathrm{keV})$ and ROSAT $(0.1 \ldots 2.0 \mathrm{keV})$ and optical data obtained during a 45-day campaign in 1994.

\section{The periods of BY Cam}

There are 4 known periods: the orbital period $(201.30 \mathrm{~m})$, the spin period of the white dwarf $(199.3303 \mathrm{~m})$, a spin-orbit beat period $(14.15 \mathrm{~d})$ and a side-band period $(197.4 \mathrm{~m})$. The detection of this side-band period lends credence to the theory of [2], who suggest that for a stream accreting, diskless, magnetic CV a frequency, $f=2 \omega_{\text {spin }}-\Omega_{\text {orb }}(=197.399 \mathrm{~m})$, will appear as a strong spike in the power spectrum for certain systems. Wu \& Mason (this volume) discuss a competing model where $P_{\text {spin }}=197.4 \mathrm{~m}$.

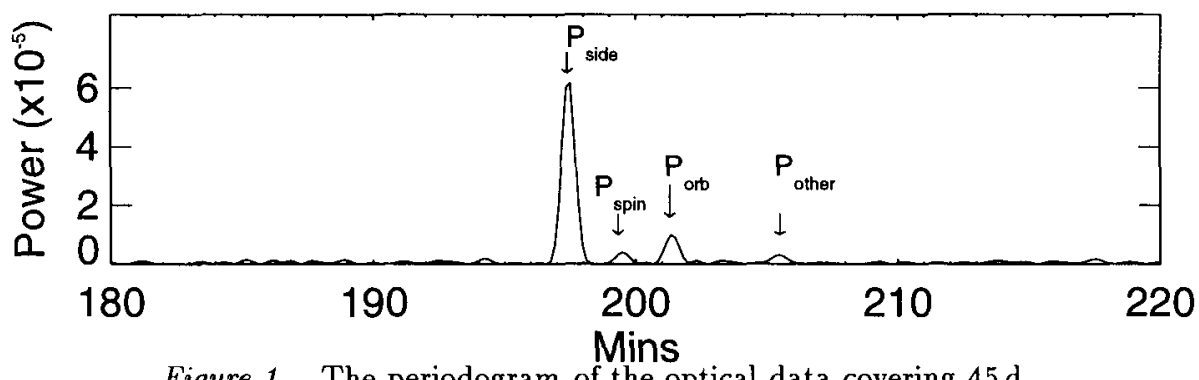

Figure 1. The periodogram of the optical data covering $45 \mathrm{~d}$.

213

A. Evans and J. H. Wood (eds.), Cataclysmic Variables and Related Objects, 213-214.

(C) 1996 Kluwer Academic Publishers. Printed in the Netherlands. 


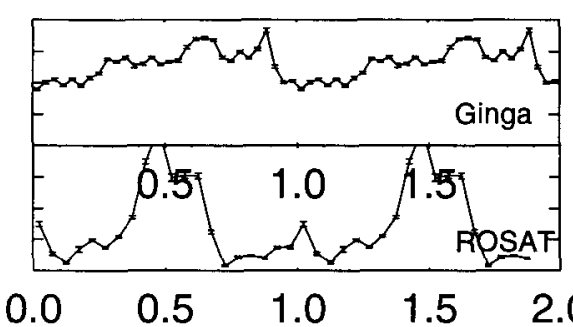

Phase

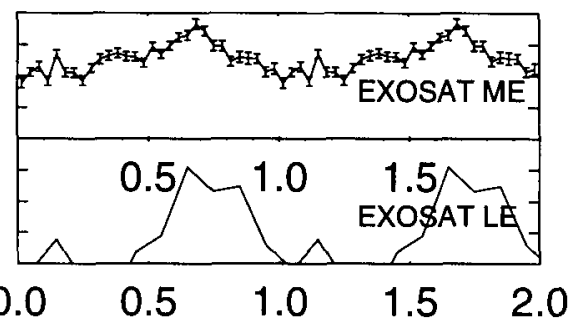

Phase

Figure 2. X-ray and optical data folded on the side-band period $\mathrm{P}=197.399 \mathrm{~m}$ as a function of phase. A proposed period derivative of $0.264 \mathrm{~s} \mathrm{yr}^{-1}$ is included.

\section{The optical data}

Fig. 1 shows the periodogram obtained from data covering a baseline of $45 \mathrm{~d}$ (see Silber, this volume). A very strong peak is seen at the side-band period, a weaker secondary peak at the orbital period and a very weak peak near the spin period. Data from a few days during which the accretion geometry is stable show the spin period, while data with a baseline of more than a few days show the side-band period with negligible power at the spin period. Another frequency, $3 \omega_{\text {spin }}-2 \Omega_{\mathrm{orb}}$, predicted by [2] is seen near $205.5 \mathrm{~m}$.

\section{X-ray data}

Periodograms of the ROSAT data show a peak at exactly twice the sideband period. Since the ROSAT baseline covers $\sim 1 / 2$ of a beat cycle we would expect a pole switch to take place during this observation and hence the spin peak would not be seen. It is likely that twice the side-band period is seen rather than the side-band period, due to the very sparse sampling inherent in the ROSAT observations. The Ginga data shows a very broad peak, but favours the $199.33 \mathrm{~m}$ spin period.

\section{The folded light curves}

To obtain phase folded light curves which can be suitably compared we need to derive the quadratic term (which is almost certainly present) with some accuracy. At this stage we tentatively propose $\dot{P}_{\text {side }}=0.264 \mathrm{~s} \mathrm{yr}^{-1}$ based on what is expected from the ephemeris of [1]. Fig. 2 suggests that the EXOSAT and Ginga data are well phased, but the ROSAT data is possibly out of phase by $\sim 0.2$ cycles. Work is in progress to refine both $P_{\text {side }}$ and $\dot{P}_{\text {side }}$ from optical and X-ray data.

\section{References}

1. Mason, P.A., Andronov, I.L., Kolesnikov, S.V., et al. 1995, in "Magnetic Cataclysmic Variables", eds D. Buckley, B. Warner, ASP Conf. Ser. 85, 493

2. Wynn G., King, A.R., 1992, MNRAS, 255, 83 